\title{
LA NARRATIVA EN LOS VIDEOJUEGOS: UN ESPACIO CULTURAL DE APRENDIZAJE SOCIOEMOCIONAL
}

Resumen. De acuerdo con las investigaciones psicopedagógicas desarrolladas en el campo de los videojuegos (Esnaola Horacek, G. 2003, 2004, 2006, 2007; Frasca, G. 1997, 1999; San Martin Alonso, A. 2006; Levis, D. 2005, 2007; Gross, B. 2000; Ferrés, J. 2000) queda demostrada su intervención en los procesos de construcción de la identidad social de los jóvenes de estos tiempos. Sostenemos que los videojuegos, en tanto objetos culturales, conllevan una narrativa lúdica particular que remite a las características propias del contexto social que las diseña. Asumimos una postura crítica y valorativa de las posibilidades educativas que brindan los videojuegos deshechando argumentos funcionalistas que los señalan como propiciadores de conductas hiperviolentas o compulsivas. La propuesta lúdica y el relato que se despliega en estos particulares entornos interactivos constituyen un espacio cultural simbólico (Bourdieu 1990) privilegiado de simulación y construcción de la dinámica de las interacciones sociales y afectivas que es preciso comprender para incorporar a las propuestas educativas.

Consideramos que es de suma importancia advertir el valor de las narrativas digitales para intervenir en ellas contribuyendo a la optimización y resguardo de la calidad de los productos que se ofrecen. Estas consideraciones brindarán orientaciones de interés para los desarrolladores, la industria de videojuegos como así también los consumidores de juegos en general.

Palabras clave: videojuegos, narrativa, aprendizaje emocional, arquetipos de identificación, espacio sociocultural, educación, teoría crítica y tecnología.

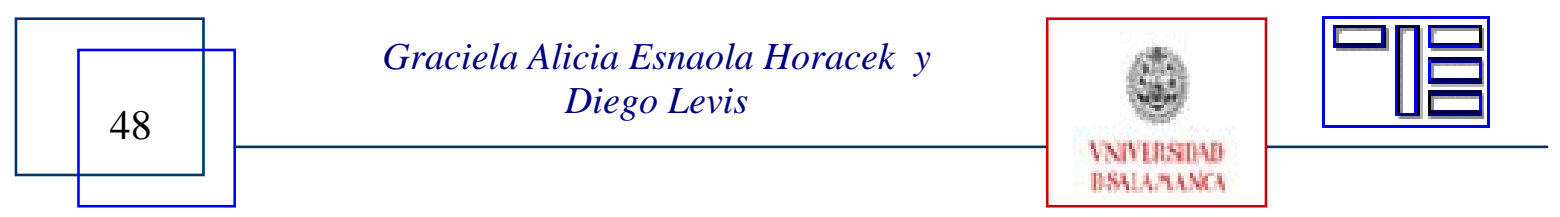




\section{STORYTELLING IN VIDEOGAMES: A CULTURAL SPACE TO LEARN EMOCIONAL HABILITIES}

Abstract. We want to analyze the characteristics of nowadays learning and the construction of the social identity through the storytelling of videogames. We recognize these technologies as an "homogeneous culture discourse" that can only be understood searching the keys that move the world beyond the walls of the school. From this perspective we are interested in the new spaces of power and authority that these technologies introduce in the institutions. To understand these processes we organize the analysis around certain aspects that operate in the interaction between children and this object. These reasons will guide us in the analyses.

The problem of our investigation is defined by stating that videogames are involved in the construction of the social identity of the users facilitating archetypes of identification models. These discourses, also, design learning modes that generate a microculture of practices and meanings with a particular logic different from the school culture.

As educators, we are interested in understanding how students organize their behaviours and identifications once immersed in this technological culture.

Furthermore, we aim at comprehending the communicative strategies developed by users when utilizing this technology. We claim that these cultural behaviours bring about consequences in academic learning.

This investigation presents basic aspects of the theoretical background, underpinning the construction of the social identity in the context of the global condition and the hybridization of cultures. We point out the characteristics of the cultural scenario linking the students and the Informational Society. It also studies in depth the characteristics of the digitalization of the narrative space and the ludic area, which offer us the possibility to analyze videogames from a complex perspective.

To sum up, our main interest is to ascertain the characteristics of learning within the storytelling and game narrative generated by videogames. We propose an educational intervention which promotes both the development of thought, and the comprehension of cultural meanings.

Keywords: videogame, narrative, ludology, emocional learning, archetypes of identification models sociocultural spaces, education, Contemporary Critical Theory and Technology.

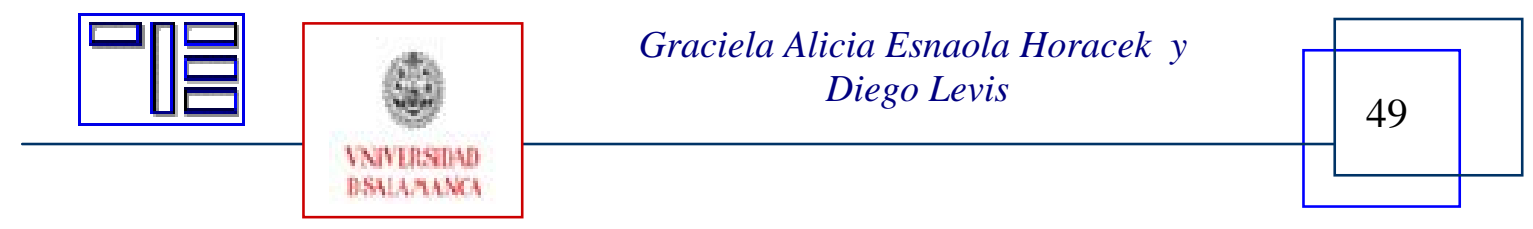




\section{LA NARRATION DES JEUX VIDÉO: UN ESPACE CULTUREL POUR L’APRENTISSAGE SOCIO-ÉMOTIONNEL}

Sommaire: Des études psicopédagogiques développés dans le domaine des jeux vidéo (Esnaola Horacek, G. 2003, 2004, 2006, 2007; Frasca, G. 1997, 1999; San Martin Alonso, A. 2006; Levis, D. 2005, 2007; Gross, B. 2000; Ferrés, J. 2000) démontrent l'intérvention de ces jeux dans les procés de construction de l'identité sociale des jeunes. On soutien que les jeux vidéo, pendant que des objets culturels, ont une narrative ludique particulière qui réflet les caractéristiques du context social dont ils appartient. On assume une position critique et de valoration des posibilités educatifs des jeux video, donc on refuse les arguments fonctionnelistes que acussent ces jeux d' inciter des comportements hiperviolents ou compulsifs. L' offre ludique et le récit que se developpent dans ces environments interactifs particuliers sont un espace culturel simbolique (Bourdieu 1990) pour la simulation et la construction des interactions sociales et afectives qu'il faut comprendre pour y incorporer aux nouvelles propositions educatives. On considere très important tenir en compte la valeur et le potentiel des narratifs numériques pour contribuer à assurer la qualité des produits. Cet article donnera des orientations sur ces sujets autant aux enseignants, a l'industrie des jeux video, aux parents et aux utilisateurs des jeux.

Mots clefs: Jeux video, narratif, apprentissage emotionel, archétypes d' identification, espace socioculturel, education, théorie critique, technologie.

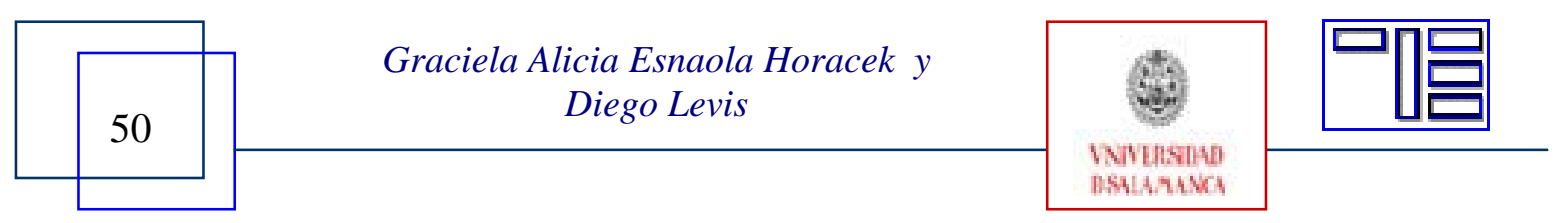




\section{LA NARRATIVA EN LOS VIDEOJUEGOS: UN ESPACIO CULTURAL DE APRENDIZAJE SOCIOEMOCIONAL}

Graciela Alicia Esnaola Horacek

graesnaola@yahoo.com.ar

Diego Levis

\section{1.- VIDEOJUEGOS Y NARRACIÓN}

Jugar y narrar son actividades cognitivas y afectivas que desde muy temprana edad posibilitan la expresión y desarrollo de la función simbólica (Piaget, Brunner) aportando contenidos y significados desde los entornos socioculturales en los cuales se sitúa el sujeto en un interjuego permanente con los significados y contenidos intrasubjetivos. De este modo, actividad y propuesta lúdico-narrativa se constituyen en factores privilegiados para la comprensión y la intervención pedagógica desde una mirada sociocrítica.

Situando el relato de los videojuegos dentro de esta línea de pensamiento y de acuerdo con las investigaciones psicopedagógicas desarrolladas en este campo (Esnaola Horacek, G. 2003, 2004, 2006, 2007; Frasca, G. 1997, 1999; San Martin Alonso, A. 2006; Levis, D. 2005, 2007; Gross, B. 2000; Ferrés, J. 2000) ha quedado suficientemente demostrada la intervención de esta modalidad de juego en los procesos de construcción de la identidad social y en los aprendizajes psicoafectivos. En tal sentido constituyen una vía regia para comprender el escenario sobre el cual el propio sujeto construye la expresión simbólica de sus preocupaciones, sueños, esperanzas y temores...

Las investigaciones que hemos coordinado, realizadas con pequeños videojugadores de tierras muy diversas nos han conducido a sistematizar rigurosamente las observaciones de niños y niñas de la Patagonia Austral y de Valencia, corroborando los indicadores significativos en otros contextos socioculturales de Argentina y España, con lo cual nuestras hipótesis iniciales se ven reafirmadas en el tránsito por las culturas de los usuarios y las propuestas lúdicas que ellos eligen para jugar. El videojuego, de acuerdo a nuestra tesis, representa el formato tecnológico que socializa a nuestros niños en la cultura lúdica ocupando el lugar que los juguetes tradicionales tenían en las generaciones anteriores. Aplicando esquemas de análisis sociosemióticos a estos entornos lúdicos es posible comprender los sentidos y modalidades de aprendizaje socioafectivo que se

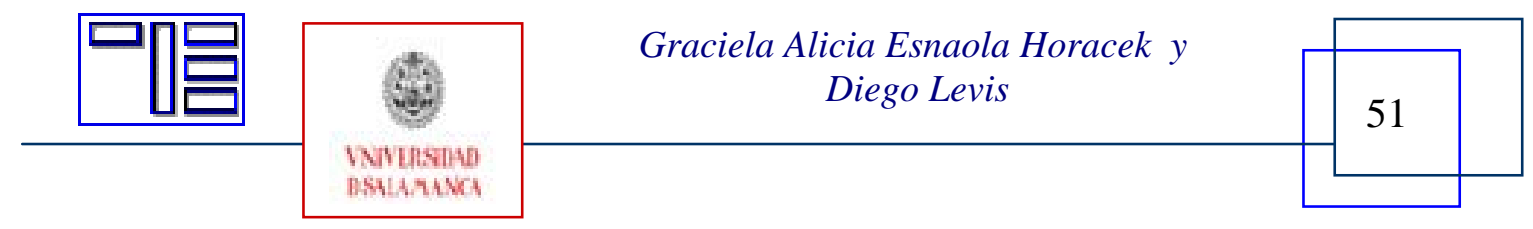


están instalando en los tiempos actuales y que pertenecen a un paradigma de construcción del conocimiento distante al propuesto desde el formato institucional escolar.

En este sentido sostenemos que los videojuegos, en tanto objetos culturales, conllevan una narrativa lúdica particular que remite a las características propias del contexto social que las diseña. Asumimos una postura crítica y valorativa de las posibilidades educativas que brindan los videojuegos deshechando argumentos funcionalistas que los señalan como facilitadores de conductas adictivas hiperviolentas o compulsivas. Desde esta mirada es que subrayamos la importancia de rescatar las claves culturales para la construcción del conocimiento en la propuesta lúdica de los videojuegos (Esnaola, 2006). La fuerte presencia de los medios masivos de comunicación y del discurso tecnológico cumplen, efectivamente, la función de evasión de una realidad que se presenta hostil, cargada de demandas y exigencias que los ciudadanos comunes, difícilmente, son capaces de abordar.

Esta realidad instalada desde los valores del neoliberalismo postmoderno provoca movimientos desubjetivantes y mecanismos de huida y de refugio en el mundo de lo virtual que se activan para preservar al psiquismo del caos. Por ello es que la narrativa de estos entornos lúdicos se presenta como un apasionante desafío para instalar la pregunta sobre el conocimiento y la construcción de una ciudadanía más comprometida socialmente en un proyecto de convivencia política signada por los valores de la paz y el bien común. Está demostrado en el conocimiento histórico experiencial que siendo espectadores pasivos de una realidad construida por otros, difícilmente se pueda activar el compromiso social y político que como generación nos compete. La propuesta lúdica y el relato que se despliega en estos particulares entornos interactivos constituyen un espacio cultural simbólico (Bourdieu, 1990) privilegiado de simulación y construcción de la dinámica de las interacciones sociales y afectivas que es preciso comprender para incorporar a las propuestas educativas.

En este sentido observamos que el paradigma epistemológico que sostiene la actual sociedad global se anuda peligrosamente en algunas propuestas didácticas a la hora de formar sujetos dóciles y reproductores de los valores que, sin embargo, en el discurso técnico-pedagógico explícito difícilmente se sostenga. Esto es, en las prácticas educativas se debería privilegiar espacios donde los participantes (estudiantes y profesores) construyan activamente la coherencia entre discursos y prácticas y se asuma la responsabilidad social en la construcción de un mundo más solidario y comprometido con la "diversidad" tanto sea cultural como ambiental. Una mirada crítica y enriquecida desde estos aportes psicopedagógicos intenta promover el diseño y la gestión de propuestas educativas que favorezcan la selección y aprovechamiento de los productos culturales de uso masivo para superar el curriculum convergente. En este sentido las propuestas lúdicas de los entornos tecnológicos debidamente seleccionadas, pueden constituirse en espacios privilegiados si son incorporados a una propuesta pedagógica renovada. En esta dirección es que ubicamos nuestras contribuciones.

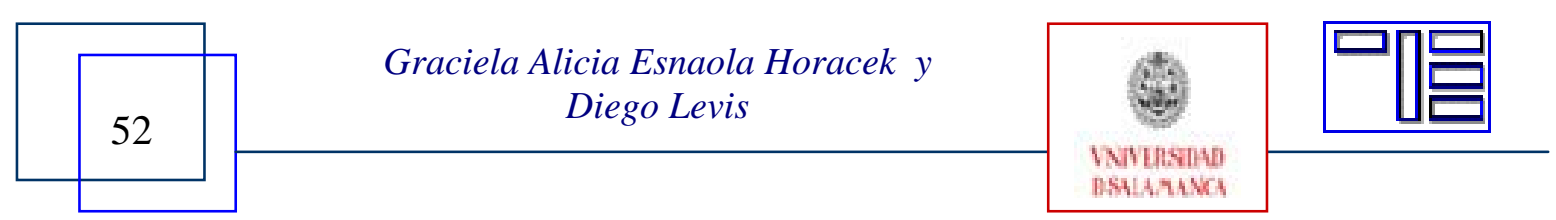




\section{2.- CONCEPTUALIZACIONES TEÓRICAS SOBRE NARRATIVA EN VIDEO- JUEGOS Y APRENDIZAJE EMOCIONAL}

"Estoy totalmente convencido de que los videojuegos pueden ayudarnos a entender mejor el mundo. Este ha sido el foco de mis estudios académicos, pero también de mis proyectos independientes. Cuando sumamos a esto la capacidad de Internet de hacer llegar tus juegos a un público internacional, empezamos a ver más y más casos de gente que crea un videojuego porque tiene algo para decir y centenares de miles de personas lo juegan. La idea de que los juegos son solamente para entretenimiento es una tontería. No digo que tengan que ser aburridos, pero los juegos pueden ir mucho más allá que el simple entretenimiento. Lo mismo sucede con el cine, la televisión, la música. Los videojuegos no tienen por qué ser excepción” (Entrevista a Frasca, M. desarrollador de videojuegos http://www.espectador.com )

Estos párrafos plasman la idea que estamos desarrollando en nuestros textos y que cada vez reúne a mayor cantidad de profesionales que consideran posible el desarrollo, producción y difusión de productos mediáticos - y en particular videojuegos- valiosos culturalmente. Para ello, es requisito primero advertir que es necesario construir un sostenido esfuerzo colectivo para instalar una lógica comercial diferente, que promueva el consumo responsable de productos de calidad. Es el tiempo del encuentro interdisciplinario entre el mundo de la educación y la industria de la comunicación y en este sentido es que hemos destacado para el análisis educativo algunas propuestas que consideramos apropiadas.

En principio debemos describir las características de los conceptos "narrativa y juego" que estamos aplicando a estos entornos particulares. Siguiendo las conceptualizaciones de algunos de los autores citados (en particular Frasca, 1999; Caillois, 1967) consideramos que es necesario entrecruzar los aportes de la "ludología” a los de la "narratología” para comprender el relato de los videojuegos en su especificidad. El concepto "juego" remite a diferentes voces en otros idiomas, conflicto que no se presenta en el castellano. Siguiendo los aportes de un autor clásico en teorías sobre el juego (Caillois, 1967) podemos definir la palabra juego (español) desde los dos términos existentes en idioma inglés: "play” y "game”.

El término "play" remite al concepto de "paideia” en referencia a una actividad derivada hacia la diversión y el entretenimiento, propia de los niños (What is done for amusement recreation). En este sentido "play" es jugar por el sólo placer de desplegar una actividad creativa, el "placer por el sólo gusto de jugar", sin ninguna meta a lograr o bien que el propio proceso es el que va definiendo los logros. Este sentido podría vincularse a las propuestas lúdicas de dibujo y diseño de escenarios, personajes o relatos creativos donde no se sigue un programa determinado y el usuario-jugador mantiene la autoría en la mayoría de sus decisiones lúdicas.

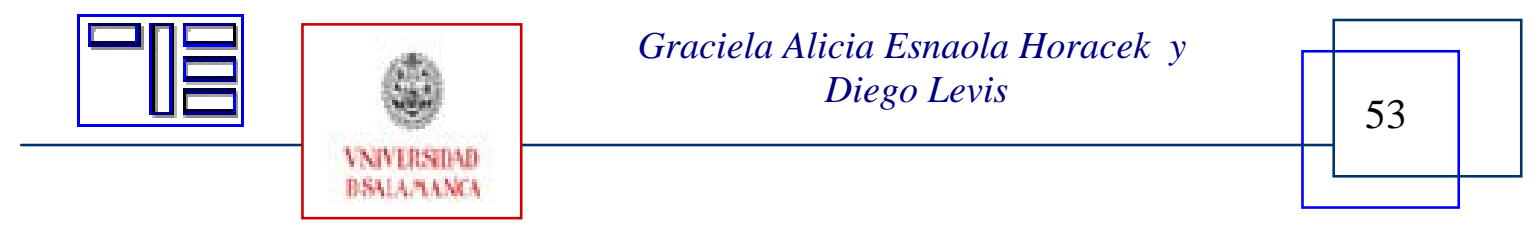


Esta acepción de juego-play es la que hallamos en las propuestas de videojuegos de simulación (las diferentes versiones de simuladores sociales: SIMS, político históricos: Age of Empires, iNo pasarán! (Guerra civil española) September 12th etc, los simuladores de Dios: Simcity, Civilization, Global Warming, etc.) y juegos de rol multiusuario (MUDS)

Sostenemos que las propuestas lúdicas de estos videojuegos son las más apropiadas para introducirse en el ámbito educativo ya que posibilitan un mayor despliegue del potencial creativo de los usuarios que llevan a cabo una autoría derivada de la propuesta de los diseñadores (Esnaola, 2006:96). Estas ventajas, además de acercarse al esquema narrativo del discurso pedagógico, conllevan otros beneficios como los de brindar la posibilidad de detener el tiempo de juego para reflexionar sobre las decisiones apropiadas, la búsqueda de información valiosa y válida para continuar el proceso, entre otras. El mayor obstáculo para su inclusión en los centros escolares -situación corroborada en las experiencias educativas estudiadas- es que estos juegos demandan mucho tiempo para su desarrollo y un despliegue de recursos tecnológicos específico que pocos centros escolares poseen, sumado a la escasa formación del profesorado en general para aprovechar las propuestas de estos entornos. Condiciones todas ellas de viabilidad para que una propuesta educativa de este tipo pueda llevarse a cabo.

En la segunda acepción del término "juego" corresponde en inglés al vocablo "game”, vinculada al concepto "ludus" que refiere a los juegos con reglas y formas determinadas, con un programa y metas a alcanzar, mas propio de los juegos de los niños de mayor edad. ("Form of play, specially with rules”)

Esta acepción pone el énfasis en el placer de dominio, de alcanzar un logro para la superación de los propios límites. En este punto hallamos coincidencias entre el proceso lúdico (en el sentido de “game- ludus”) y el proceso narrativo (Bremond, 1970).

En ambas estructuras el proceso sigue una estructura lineal que se inicia en un estado de equilibrio (begining) interrumpida ante una situación de conflicto que lleva al lectorjugador a tener que optar por alguna salida posible para alcanzar el triunfo o asumir la derrota. (con el consiguiente reinicio del proceso)

Este esquema es el que advertimos en los videojuegos de aventuras gráficas, los arcade o los de competencia, en general. Son propuestas lúdicas con un esquema narrativo básico, cuyo desafío está instalado en la respuesta apropiada y ajustada al éxito en el programa preestablecido. El jugador tiene que adquirir la destreza suficiente para responder adecuadamente en un tiempo cada vez más breve y complejo a fin de alcanzar estándares superadores del ranking alcanzado por otros usuarios.

El componente de destreza técnica y desarrollo de habilidades propias del entorno de alta competencia es un factor importante en el logro de rankings de competitividad propios de determinados niveles de exigencia en la performance de los sujetos. Algo simi-

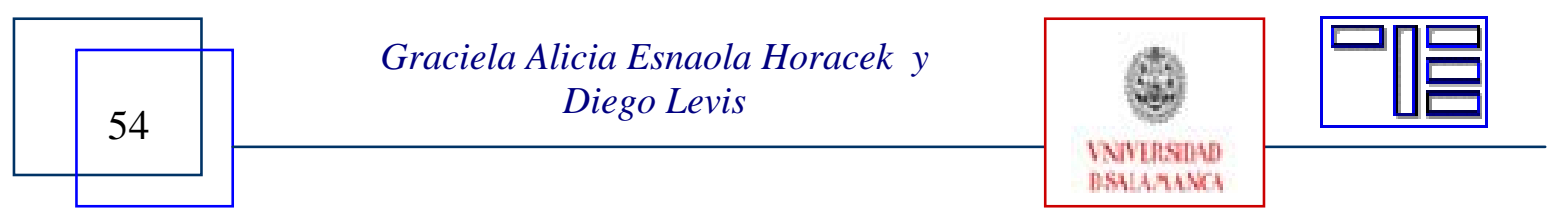


lar a lo que se pretende alcanzar desde las prácticas escolares cuando incluimos olimpiadas y competencias de rankings y estándares en los que se premian los mejores y se descartan los menos hábiles.

En ambas acepciones del término "juego" hallamos diferentes propuestas narrativas que facilitan el despliegue de competencias cognitivas y socioafectivas diferentes y, en cierto sentido, complementarias. Estas características deberían ser consideradas al momento de seleccionar o evaluar la pertinencia de incluir un videojuego con fines educativos.

\section{3.- PROPIEDADES DE LOS ENTORNOS DIGITALES Y LA NARRATIVA}

Para comprender la lógica narrativa específica de los entornos digitales es preciso advertir que introducen una ruptura en la percepción que no es la admitida en las perspectivas espacio temporales en las que es necesario fragmentar el tiempo para percibir la totalidad. En los entornos digitales -cuya principal característica es la inmersión participativa, la actuación y la transformación (Esnaola, 2006)- ha desaparecido el transcurrir temporal para situarse en la percepción de la acción en el presente del narrador.

En las narrativas hipermedia, a diferencia de las narrativas secuenciales, estas instancias se alteran por las características propias del entorno participativo que diluye las fronteras entre el narrador, el personaje y el propio lector. El ámbito del discurso electrónico impone sus propias reglas al relato que debe construirse a partir de la fragmentación en la superficie discursiva. En estos entornos los saberes del narrador y del personaje emergen de la fractura del espacio dramático y se dispersa la percepción de lo narrado bajo el predominio de una nueva perspectiva.

Desde nuestro marco referencial consideramos que estos entornos propician un proceso narrativo particular que se caracteriza por la apertura de un espacio virtual tradicional (Winnicott, 1982) entre el narrador y su historia que provoca al lector para que participe en el proceso de elección de las secuencias narrativas. Encontramos entonces que estos procesos son considerados interactivos en su especificidad ya que emplean a la vez dos estrategias complementarias: son sucesivos y participativos.

\section{4.- CARACTERÍSTICAS DE LA NARRATIVA DIGITAL EN EL CASO DE LOS VIDEOJUEGOS}

Hemos descripto a las historias multiformes como obras escritas o dramatizadas que presentan una única situación o argumento en múltiples versiones mutuamente excluyentes (Murray, 1999) en la experiencia temporal cotidiana. Estas narraciones buscan mostrar las diferentes posibilidades que se nos presentan en cada momento de decisión. Pero para que el autor pudiese desarrollar estas distintas perspectivas es necesario contar con un medio tecnológico capaz de brindar esas posibilidades. El desarrollo de la tecnología digital específicamente en el caso de los videojuegos ha demostrado sus amplias posibilidades para el despegue narrativo de historias de múltiples posibilidades en las

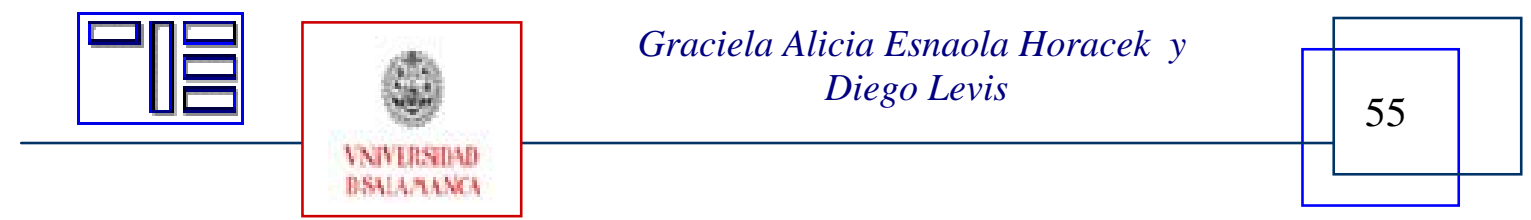


que se invita permanentemente al lector-jugador-narrador a realizar él mismo las opciones narrativas. En este mundo de cambios tan vertiginosos e inestables, el narrador ha quedado desconcertado, enredado en las múltiples relaciones y posibilidades que se van abriendo ante su imaginación creadora. Si el narrador se ciñe a una determinada historia -tal es el caso de los relatos con argumentos lineales tradicionales- estaría realizando una opción dejando de lado a las múltiples posibilidades que existían en el momento previo a su elección. Es entonces que encuentra propicia la estrategia de narrar participativamente con el propio lector. Al invitarlo a implicarse en el proceso de elección de secuencias, el narrador abre un espacio transicional que le permite al lector participar en la creación narrativa. Al participar en las elecciones de la historia, el lector se "descentra” y se ubica "como si” él mismo estuviese creando la historia. En el caso de las narrativas de los videojuegos con propuestas como las citadas anteriormente como "play paideia" podemos comprender los movimientos de implicación cognitivo afectivo que suscita este proceso de inmersión en la trama y entender la concentración e interés que despiertan -entre otras competencias en juego- y deshechar el argumento de la "falta de concentración" en los usuarios, precisamente porque el cuantum de energía psicoafectiva requerida es muy importante para desplegar la propuesta lúdica.

Las narraciones de novelas o películas de misterio han empleado con mucha eficacia esta estrategia provocando en el lector su implicación en la trama, mucho más aún si él conoce las propiedades del género de suspense. Es también un elemento muy bien utilizado por los productos en serie ya que sostienen la atención del lector/espectador en torno a las diferentes posibilidades en las que la historia se desarrollará y continuará... después de una pausa provocada para mantener en suspenso la acción. Estos productos narrativos seriales conforman la estructura básica de las entregas periódicas de las revistas de historietas, las series televisivas o las entregas de las diferentes versiones de los juegos electrónicos. Hallamos entonces que esta estrategia, al implicar al lector, convoca a la audiencia trastocando su lugar en el "espectáculo", un lector, una audiencia que es convocada a adoptar una postura activa, componiendo los fragmentos de la historia y concibiendo trayectorias más o menos disímiles entre ellas.

Afirmamos entonces que las nuevas tecnologías pueden llegar a brindar amplias posibilidades para narrar historias siempre que se respeten las reglas que propone su juego, de la misma manera que podemos leer en sus relatos cómo han quedado inscriptas las convenciones sociales de la época para comprender el mundo de la cultura que las sostiene. Nuestro desafío radica entonces en comprender sus códigos, aprender a leerlos y a contar historias coherentes utilizando un medio tan cambiante como nuestros propios tiempos.

Para comenzar a desentrañar esta "literatura de la transformación” es necesario establecer las nuevas convenciones narrativas que nos permitan la inmersión en este escenario para actuar en él narrando nuestros significados.

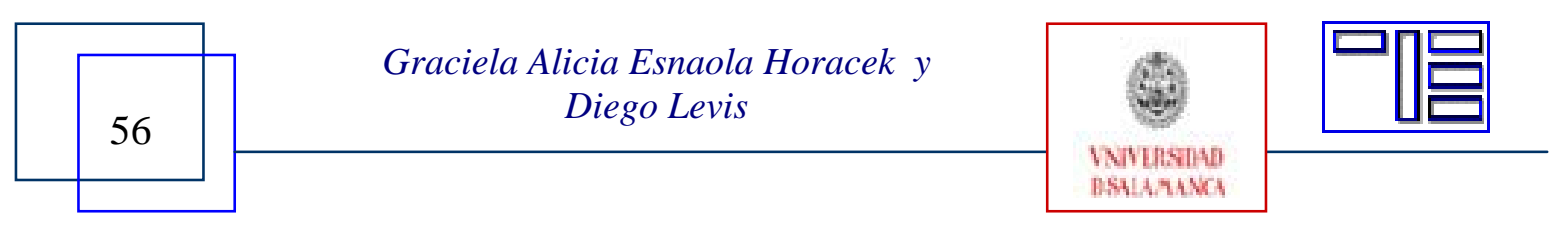


Marshall McLuhan describió a los medios de comunicación del siglo XX como una estructura en forma de mosaico en contraposición a la estructura lineal del libro impreso. Estos medios fueron presentándonos la información en formatos fragmentados, diseminados en las superficies de periódicos y textos impresos que poco a poco fueron superando el impacto inicial que provocaron en el proceso lector acostumbrado a la continuidad y a las superficies extensas y monocromáticas. A este universo se sumó el impacto en los medios audiovisuales, el cine y la TV, que incluyeron composiciones narrativas realizadas en base a múltiples enfoques particulares. A la fragmentación de las superficies se sumó, entonces, la dispersión del narrador. El medio-mosaico fue presentándose ante el lector que poco a poco se inició en esta lectura fragmentaria y en el pensamiento-mosaico de modo tal que en estos momentos prácticamente estamos comenzando a superar el desconcierto inicial. Y todo esto se produce en parte por la frecuencia de las lecturas fragmentadas que han instalado el predominio del hipertexto y la superposición de historias en paralelo a través del tiempo y los escenarios.

Nuestra percepción de múltiples mensajes ha aprendido a realizar una lectura rápida, superficial, que rápidamente busca los puntos de interés basándose en las huellas que el diseño gráfico le ha mostrado: letras más grandes, resaltadas, en primera plana, titulares más importantes en el extremo superior derecho y así sucesiva y jerárquicamente se acompaña el barrido que la mirada acostumbra a hacer siguiendo las normas de nuestro alfabeto occidental. Hemos aprendido también a intuir la estructura a partir de imágenes discontinuas y hasta podemos avizorar una nueva categoría de placer estético en el uso del zapping, en una búsqueda por yuxtaponer escenas o episodios de acuerdo a nuestro propio interés como usuarios.

Con el advenimiento de los ordenadores la metáfora del mosaico de Mc Luhan se reformula en la narrativa calidoscópica propuesta por Murray, en tanto las posibilidades particulares del multimedia interactivo reúnen el mosaico espacial de los medios impresos, el mosaico temporal del cine y el mosaico participativo del mando a distancia del televisor.

Esta metáfora del calidoscopio nos remite a una idea de las múltiples posibilidades que se pueden obtener a partir de lo fragmentario en una suerte de transformación de las fronteras entre las narraciones y los juegos, entre las formas narrativas y las formas dramáticas, desde la audiencia pasiva a la participativa. Esta posibilidad que permite el medio de acudir a distintas acciones simultáneas narradas de diferente forma, propone entonces un nuevo género narrativo que reúne las posibilidades narrativas y dramáticas en un mayor despliegue de efectos provocando la inmersión participativa del lector (devenido actor-coautor) en la trama. De modo tal que la obra se desarrolla en un entorno mucho más creíble favoreciendo la identificación del lector con el narrador en el relato. Estas historias que remiten a múltiples líneas paralelas o entrecruzadas favorecen el desarrollo de una lectura que incluye diferentes puntos de vista y que nos invita a pensar incluyendo lo diverso, lo múltiple, lo complejo. (Morin, 2000)

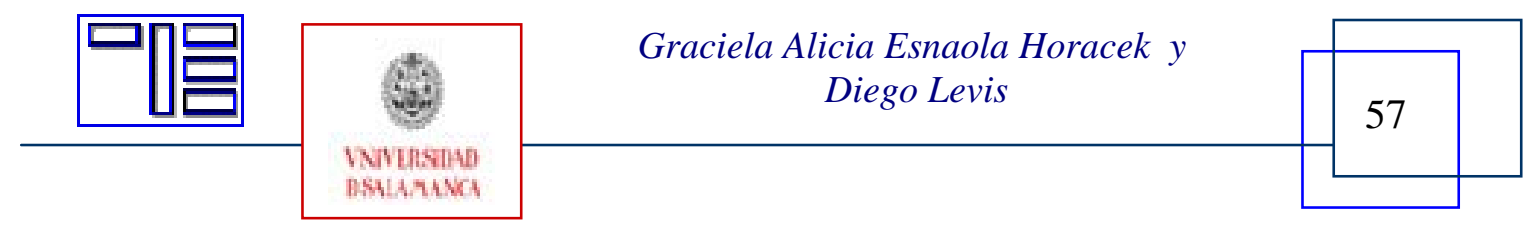


Pero estas posibilidades requieren que determinadas convenciones entren en el juego narrativo a fin de no perder en la fragmentación del medio, la coherencia de la historia provocando la saturación del lector. La propuesta narrativa debe provocar la búsqueda del argumento a través de los escenarios, despertar el interés del lector por salir de la escena y explorar otros lugares posibles sin disgregar la historia. En este punto radica la importancia de establecer convenciones propias del medio. Las historias que narran en un mismo momento distintas miradas desde diferentes puntos de vista nos pueden mostrar los planos emocionales diversos que convergen en una misma situación.

De esta manera aumentamos nuestra capacidad de percepción poniéndonos en los lugares de los otros, descentrarnos como lectores que observan el transcurrir de la historia para sumergirnos como actores implicados en la situación. Esta nueva posibilidad nos ubica en un lugar que no se corresponde con las categorías narrativas tradicionales, un momento de iluminación dramática que nos permite la identificación con los personajes y el transcurrir de los relatos particulares en una conjunción entrelazada desde nuestra inmersión en la narración. Nos permite captar los fragmentos del calidoscopio pero para conformar con ellos una experiencia estética. En este punto encontramos cómo las posibilidades del medio nos relatan nuestra percepción del mundo y de sus significados. Percibimos que ninguna posición puede albergar todos los significados y que tampoco una sola mirada puede constituirse en un relato totalizador de la realidad, diseminada en las voces múltiples y diversas pero que encierran en sí mismas la coherencia del significado.

Las narrativas calidoscópicas nos permiten visualizar las posibilidades del medio más allá de la producción digital que nos invade diariamente y que aprovecha sus características fascinantes y enciclopédicas para distribuir sus productos en búsqueda de réditos económicos, en desmedro del desarrollo de sus posibilidades.

En la discontinuidad de los procesos tecnológicos podemos encontrar lo propio y valioso que puede ofrecer cada nuevo entorno narrativo. Las novelas o historias desarrolladas bajo el predominio del texto impreso nos permiten explorar el mundo de los personajes; las obras dramáticas nos permiten inmiscuirnos en las características de la acción. La narrativa de simulación, en cambio, posibilita nuestra inmersión en el proceso narrativo, adquiriendo la condición de cosa a experimentar que de algún modo funde la narración a la vida, en tanto los hechos que se suceden en el juego son consecuencia de las acciones y decisiones del jugador.

\section{5.- PRINCIPIOS ESTÉTICOS DE LA NARRATIVA DIGITAL}

A lo largo de estas páginas hemos procurado profundizar la reflexión en torno a la capacidad narrativa, intrínsecamente humana, que nos permite expresar los contenidos simbólicos de nuestro mundo interno posibilitándonos la proyección de nuestros miedos básicos en objetos externos, manipulables. Al ubicar en un contexto ajeno, extrínseco, a nuestras incertidumbres, se nos abre la posibilidad de tomar distancia emocional y, de

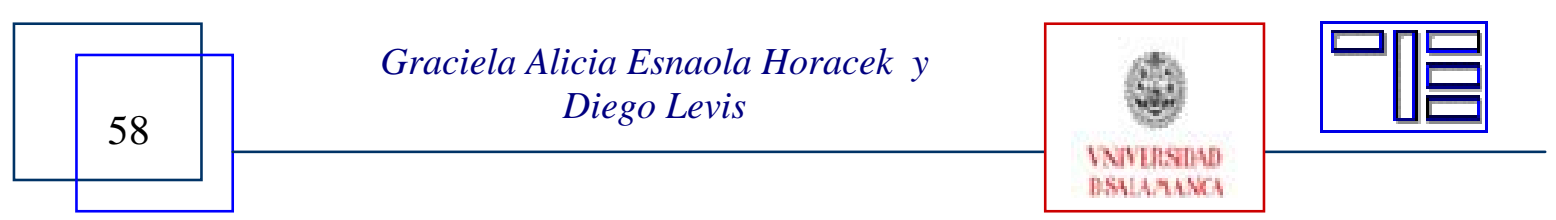


alguna manera, controlarlas sin caer en el temor a padecer riesgos. Es más fácil odiar al villano y verle vencido a manos del héroe que llegar a visualizar que pocas veces nuestro héroe interno resulta invencible y mucho menos que detrás de las mejores intenciones también hay lugar para las codicias villanas. Es mucho más difícil pasar de la fantasía en la que podemos separar claramente los personajes totalmente malos y totalmente buenos a una realidad en la que estas consideraciones presentan luces y sombras.

Este espacio de transición entre la realidad interna y la externa al sujeto utiliza determinados vehículos emocionales especialmente propicios para generarlos. En los primeros años de vida esta función transicional se traslada hacia los juguetes: objetos que el medio cultural les acerca a los niños y que ellos invisten de contenidos emocionales propios. Cada niño establece con algún objeto en particular este proceso de vinculación transicional otorgándole a la situación lúdica y al objeto sobre el cual ejerce esta función significaciones propias, asumiendo distintas posiciones respecto a él que le permiten representar sus incertidumbres hasta tanto pueda asimilarlas y controlarlas. Una vez que esto sucede, el objeto pierde su valor y cae en desuso (Winnicot, 1982).

Las narraciones, una vez instalado el lenguaje y con él la posibilidad de simbolizar mediante relatos los significados, temores y dudas, se constituyen en objetos transicionales. Narrar, darle una historia a lo innombrable nos permite el ejercicio de la función simbólica que necesita construir canales expresivos a través de los cuales darle voz a lo caótico y colocarlo bajo las convenciones que, mediante el orden del lenguaje, hemos construido en cada estilo.

Esta posibilidad de representar el mundo interno se desarrolla a través de diferentes herramientas culturales. Las narraciones literarias escritas en formato de novela nos permiten explorar las características de los personajes, su mundo interno y sus posiciones a lo largo del desarrollo de la historia, las narraciones escenificadas, en cambio, nos permiten explorar especialmente las características de la acción y los movimientos de los personajes en la escena de acuerdo con las convenciones dramáticas. En las diversas modalidades sin embargo, se conserva esta posibilidad de establecer una suerte de frontera entre el espacio del sujeto y de la obra: la constitución de un espacio de transición que nos permite jugar con nuestras propias fantasías con la seguridad de que contamos con "la cuarta pared" que nos mantiene fuera de la escena. Esta convención dramática es el punto sobre el cual la narrativa digital comienza a abrirse paso provocando la “iluminación dramática” de la escena. Mediante la narrativa participativa nuestro lugar de espectadores que pueden identificarse con lo que le sucede a los personajes, las fantasías de amor y de odio que desarrollamos sobre los héroes y los villanos, pero relatadas por un narrador externo a nuestra propia voz queda paulatinamente destruida. Hemos comenzado a des-ilusionarnos entrando en la escena, perforando la "cuarta pared del escenario". En una narrativa de inmersión dramática las emociones y las fantasías más arcaicas aparecen iluminadas con una crudeza tal que nos descontrola. La fantasía oculta bajo la identificación con el narrador queda diluida y podemos implicarnos en el mundo de la ficción que nosotros mismos hemos construido.

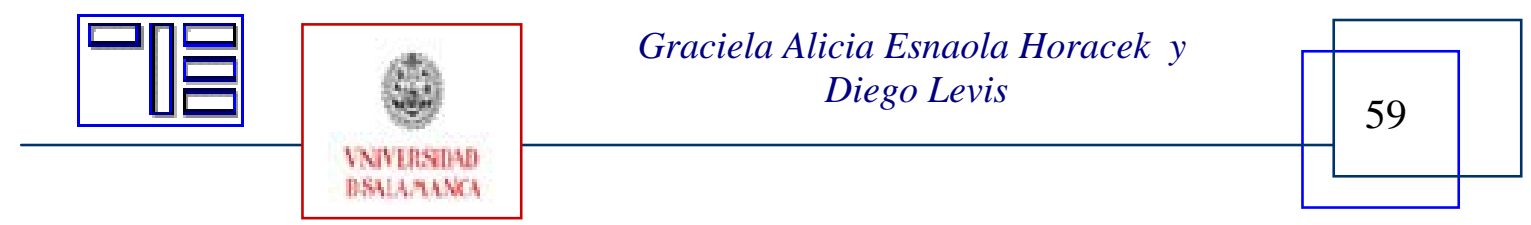


Esta característica propia de las narrativas digitales, la inmersión, es el primer aspecto que nos atrae de estos medios. La inmersión establece con el usuario una suerte de "seducción” entre lo que ofrece y lo que oculta reclamando la implicación del sujeto para avanzar en el despliegue de sus posibilidades. La experiencia de poder sumergirse en la fantasía, trasladándose a un lugar de ficción alejado de la monotonía cotidiana es un deseo que nos ha acompañado desde tiempos remotos y que ha sido visto como peligroso, con un poder de seducción capaz de provocar la escisión de la personalidad.

Este deseo ancestral de sumergirnos en la fantasía se intensifica en los medios digitales que proponen una narrativa participativa, el medio promete satisfacer el deseo de sustituir el mundo real por la fantasía. Cuando un medio atrapa el interés sumergiéndonos en la ilusión se ubica en el umbral entre la realidad exterior y la propia mente del lector. Estas experiencias en las que "lo real es lo que no está allí” (Winnicott, 1971) se prolongan en las distintas formas narrativas, desde la oralidad a la escritura estableciendo convenciones que sostienen ese espacio de transición, el frágil umbral que sostenga que “el mundo virtual siga siendo real manteniéndolo fuera de allí”.

Desde esta convención hallamos también un cauce para nuestros propios temores que permanecen en el exterior, en el espacio de la escena. La posibilidad de que afloren dichas sensaciones con total crudeza y descontrol nos produce desagrado, preferimos que lo imaginario se mantenga fuera del "umbral”.

Las narrativas participativas instalan básicamente estas preguntas ¿Cuál es el límite que debemos establecer a fin de reservar las fantasías al espacio virtual sin temer que se encarnen? ¿Cómo emplear un medio participativo sin que no nos paralicemos al sumergirnos ante nuestras propias fantasías?

Encontramos que muchos de los prejuicios que despiertan las narrativas, cada vez que avanzan en sus formatos, se dirigen en este sentido que explican las "demonizaciones" de los seductores entornos digitales de internet o los videojuegos. En particular estos temores se concentran alrededor de los efectos que pueden provocar en los usuarios y esto lo pudimos constatar en los trabajos de investigación sobre estas tecnologías. Haciendo un seguimiento de los intereses de los investigadores podemos inferir también cuáles son los temores que despiertan estas tecnologías en la comunidad científica.

¿Será quizá esta preocupación por encontrar en estos relatos una semblanza descarnada de nuestras propias posibilidades de destrucción? En una narrativa participativa que nos sumerge en la trama ¿qué caminos debemos señalar para no derribar la cuarta pared y sostener la frontera entre lo virtual y lo real? ¿Cómo sumergirnos en este espacio narrativo sin diluirnos y llegar a crear un entorno lúdico de interjuego entre la realidad y la fantasía?

Janet Murray propone estructurar la participación “como una visita”. Esta figura parece apropiada porque conlleva la posibilidad de establecer un recorte en el tiempo y el espa-

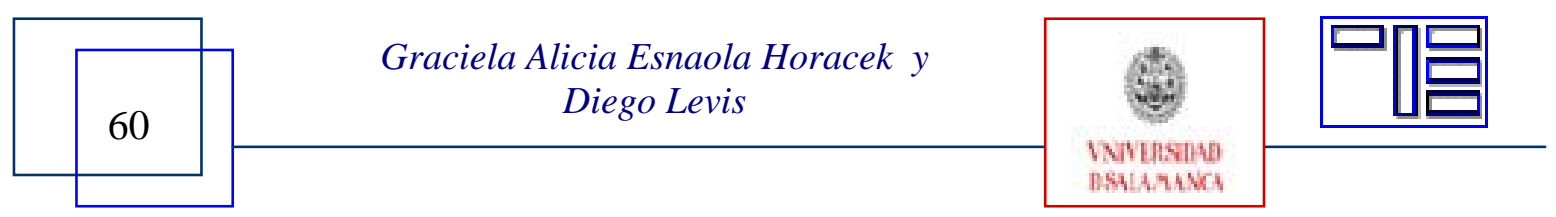


cio que establezca una suerte de frontera flexible, que señale la entrada y la salida de la escena. Un ejemplo gráfico podemos tomarlo de una visita a un parque temático. Allí tenemos un punto de entrada y otro de salida, con un recorrido que no está rígidamente seleccionado, que permite la inmersión del "navegante” pero que también le posibilita la salida de la fantasía. Permite experimentar múltiples sensaciones, establecer un juego lúdico entre el drama y la tragedia, posicionarnos desde algún personaje pero comprendiendo el "como si” de la escena participativa. Si el proceso de inmersión está bien logrado, estimula la participación activa en su trama narrativa. El segundo principio que brinda especial placer en estos entornos, que se deriva de la inmersión es la actuación. Este concepto excede a la sola respuesta de un ícono activado mediante nuestro click. La actuación implica poder llevar a cabo acciones significativas y ver los resultados de nuestras decisiones y elecciones (Murray, 1997) implica actuar dentro de un entorno narrativo. En este punto nos ubicamos en una nueva posición narrativa. Las formas tradicionales emplean la participación del lector en ocasiones muy limitadas y ya previamente establecidas en cada género. Incluso en las obras dramáticas en las que se incluye la participación de la audiencia, su lugar, si bien es invitada a realizar o responder a determinada propuesta, sigue en su lugar de auditorio y la historia sigue su curso indefectiblemente aún con estos "toques” de participación. Los entornos electrónicos también pueden presentar diferentes grados de participación. No podemos comparar un juego en el que la participación del usuario se reduce a seguir las órdenes diseñadas en el menú con un entorno de simulación en el cual, a partir de las elecciones realizadas por el usuario, los componentes del medio pueden modificarse y mostrarnos dinámicamente las consecuencias de nuestras elecciones. En este punto es importante discernir entre "interactividad" (término aplicado con demasiada amplitud) con la actuación como placer estético, aún cuando estemos refiriéndonos a conductas y resultados. La estructura lúdica de la actuación no implica cuánta actividad estamos desarrollando sino el grado de autonomía posible. Comparemos, por ejemplo un juego de ajedrez con un juego pinball: aun con la cantidad de respuestas que se dan en el segundo caso, no podemos inferir por eso que el jugador obtenga una experiencia valiosa de su actuación, como sucede en el primer caso.

La narrativa en entornos participativos que convoca la actuación del usuario puede también despertar placer estético, siempre que se aleje del repiqueteo fugaz de las propuestas de estímulo-respuesta propio de gran parte de los juegos electrónicos. Una posibilidad narrativa que ofrecen estos medios se basa en la posibilidad que brindan al usuario por "explorar el espacio", navegar por las distintas escenas, plataformas o páginas web por el sólo placer de recorrerlas independientemente del contenido de la historia. Explorar es una propuesta lúdica que desarrollamos tanto en los ámbitos reales como en los virtuales y que despiertan nuestro interés por inmiscuirnos en lo desconocido o profundizar en lo conocido. En esta exploración se requieren señales de orientación que nos permiten trazarnos caminos a través de los distintos escenarios. Las narrativas digitales toman esta característica placentera y desarrollan básicamente dos estrategias: el laberinto para resolver y el rizoma enmarañado (Murray, 1997)

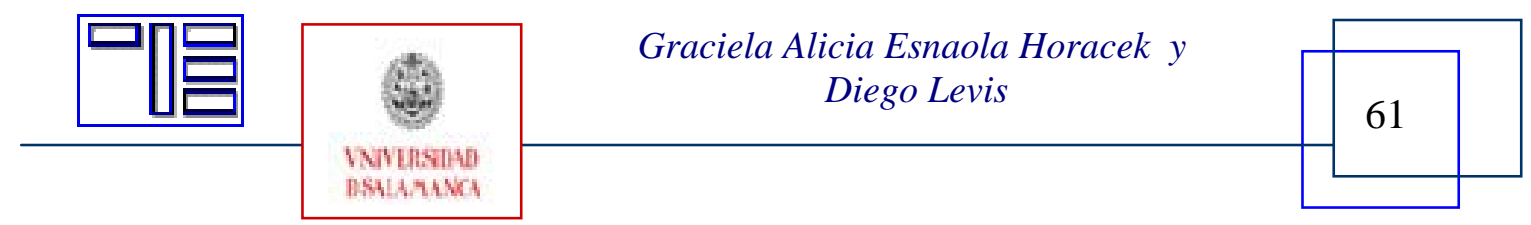


Ambas estrategias se encuentran desarrolladas desde las primeras formas narrativas heroicas en las que la historia se desplegaba a través de distintos escenarios con senderos laberínticos y peligros acechando inesperadamente. Esta metáfora ha quedado plasmada en los laberintos que tan frecuentemente hallamos rodeando los castillos medievales, por ejemplo.

Estas formas narrativas despiertan nuestros propios deseos por desentrañar los sentidos ocultos a nuestra percepción. La realidad se nos presenta como un desafío, con muy pocas certezas y con inesperados riesgos. El camino laberíntico convoca a nuestro héroe interior para lograr vencerse a sí mismo y encontrar el tesoro perdido, la princesa o el príncipe de sus sueños o sencillamente el placer de disfrutar de nuestra propia fantasía.

Esta estrategia es utilizada con mayor o menor grado de despliegue técnico por la mayoría de los videojuegos que convocan el interés de los jugadores. En muchos casos el despliegue exagerado de los efectos y la convocatoria a la respuesta acelerada del usuario que le permite vivir o matar -y ganar puntaje- detiene el desarrollo de una historia narrativa y buscan provocar la tensión y el deseo de competir del usuario en desmedro del placer de la exploración que el entorno podría ofrecer. La estructura laberíntica, en definitiva, se halla predeterminada, hay un camino que es el propuesto y que es preciso descubrir. Otra posibilidad es la estructura de rizoma en la que la ficción está indeterminada a la manera de la narrativa hipertextual posmoderna. Esta propuesta no se basa en un tipo de racionalismo griego sino en un pensamiento más propio de la teoría literaria postestructuralista. Deleuze (1987) propone esta figura del rizoma para nombrar la estructura en la que un punto puede llegar a conectarse con cualquier otro sin reconocer un cierre. Esta figura es tomada por la teoría literaria para ser aplicada a los textos que no siguen una estructura lineal, propia de los textos impresos sino que se acercan más a una frontera sin entrada o salida fija. Esta estructura apuesta básicamente a la libre interpretación del lector en desmedro de la intención del autor y renuncia a la clausura.

Por tanto la estructura laberíntica como la de rizoma son formas predeterminadas que impiden desarrollar el placer de la exploración del usuario. Las narraciones deberían presentarse en un punto intermedio: que brinden orientación al navegante pero que permanezca una estructura abierta que posibilite la exploración en el espacio narrativo del usuario. Las estructuras laberínticas generan angustia, desconcierto y temor a perder lo propio ante lo desconocido. La angustia de la incertidumbre se desplaza por el laberinto narrativo y genera tensión en el jugador. Si a esta estructura lineal se la enriquece con la posibilidad de visualizar la historia desde múltiples puntos de vista esta forma sería tranquilizadora porque brindaría mayor espacio para visualizar la situación desde múltiples versiones. Las estructuras multilineales nos permiten expresar dramáticamente nuestros miedos y narrarlos a modo de una exploración.

Esta situación de actuación narrativa nos remite a cuestiones relativas a la autoría. Existen autores que sostienen que el usuario de las narrativas digitales es el autor de la obra. Esta proposición, al igual que la referencia a la interactividad de los ordenadores es en-

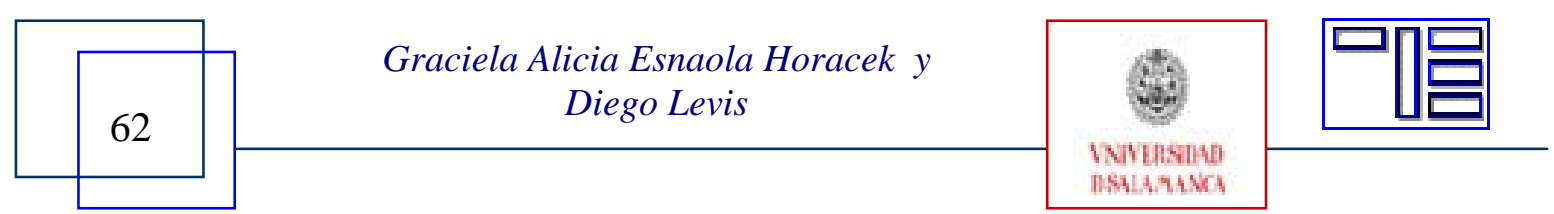


gañosa. En todo caso el usuario es quien desempeña un papel activo, elige personajes o acciones pero siempre bajo un entorno ya determinado previamente. La autoría del usuario es una "autoría derivada" de la propuesta del medio que se basa en una sucesión de procedimientos establecidos en el programa. Si bien el usuario puede modelar acciones y experimentar el placer de emplear, yuxtaponer o vincular materiales expresivos en todo caso estamos hablando de "actuación”. En este caso estaríamos estableciendo una cuestión semejante a las cuestiones de autoría de los poetas orales de la antigüedad, en los que hoy podemos rastrear su obra como fruto de yuxtaposiciones y mezclas producidas en el seno de la comunidad de origen.

La autoría en las narrativas digitales, en cambio, remite a un autor que "escribe las reglas que determinarán el texto además de escribir el texto en sí”. El autor determina las reglas de juego sobre las cuales el usuario desplegará su actuación a la manera de un director de orquesta que determina los acordes con los cuales se desarrollará la obra. Otra de las posibilidades del medio es que ofrece el placer de la transformación que nos permite adoptar múltiples personajes y cambiar de apariencia ocultando nuestra identidad. En el caso de los chats, por ejemplo, esta característica es empleada a punto tal que el código de uso es precisamente el engaño y el "todo vale". Los miembros que conversan utilizando el chat se adhieren a esta convención, entran en el juego de suprimir la propia identidad, el medio fragmenta los diálogos imprimiéndoles la velocidad y fugacidad propias de la conversación oral y transforma la escritura en una "oralidad escrita" (Levis, 2006) si es que se acepta este término. No se trata de una conversación al estilo tradicional, la máscara y la simulación constituyen el pacto inicial del acto comunicativo.

El ejemplo del chat es ilustrativo de las posibilidades que este medio ofrece a los usuarios. Un juego de seducción y de permanente mutación requiere de nuevas convenciones que le den forma a una "literatura de la transformación". Hemos descripto a este tipo de narrativa como "calidoscópica" y es fiel reflejo de nuestro pensamiento entre milenios que se halla en una búsqueda por reunir los fragmentos de verdad dispersos en un relato coherente que pueda ser capaz de albergar la diversidad y no monopolizarla.

También los argumentos desarrollados en las narrativas digitales deben avanzar hacia un nivel de expresividad más adulto. De Brontë en su obra Tales of the islanders (1911) realiza una semblanza de un tipo de literatura de transformación en la que los personajes optan por cambiar las situaciones dramáticas de sus muñecas a prototipos de mujeres con las cuales poder identificarse. Las narraciones cumplen precisamente esa función de anticiparse a los roles adultos, representándolos en los juegos o en las narraciones. Muchos de los temas que hallamos en las narrativas digitales actuales tienen esa reminiscencia adolescente de ruptura de códigos sociales tradicionales, alta carga de erotismo y de violencia que nos hablan de una carga emocional aún no encauzada que busca mostrar los conflictos y escapar de ellos sin buscar propuestas superadoras: mostrar y enunciar, ver y denunciar sin intentar comprender.

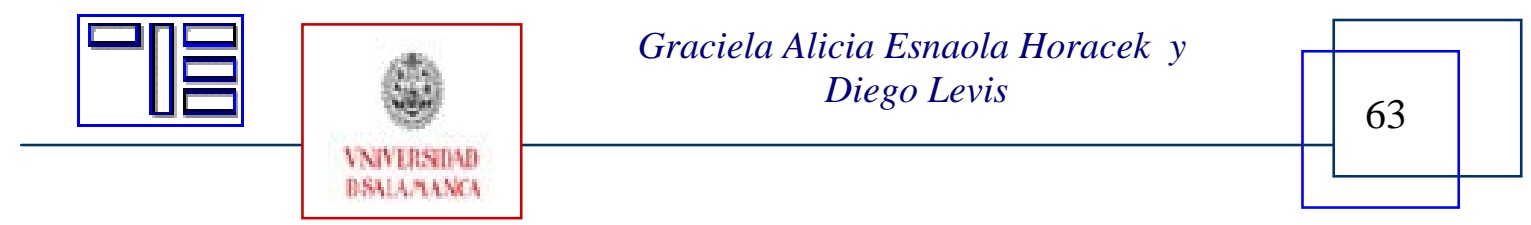


Para que las narrativas electrónicas avancen hacia mayores niveles de expresividad es preciso que las transformaciones que se producen al interior de los procesos superen las fantasías adolescentes descontroladas y comiencen a plantearse otras posibilidades que superen la repetición de temas traumáticos. Un buen indicio en estas narrativas no debería evadir los temas que nos preocupan sino incluirlos para aprovechar la posibilidad de estas narrativas calidoscópicas para visualizar el problema desde diversos puntos de vista.

La transformación que enunciamos se visualiza claramente en los ejercicios de simulación que permiten aprender a apreciar la riqueza de una narrativa calidoscópica que no necesariamente implica renunciar a la conclusión o al desahogo emocional. En la simulación podemos entrar a la trama y experimentar las sensaciones repetidamente, podemos posicionarnos desde distintos personajes y proponer distintos finales.

Estas posibilidades del medio nos permiten visualizar los procesos y nos invita a construir nuevos parámetros del placer estético que puedan incluir la complejidad. Este es un nuevo aprendizaje que nos propone el medio, tal como en su momento ha sido aprender a visualizar los códigos del movimiento en la escena dramática.

Ciertamente tanto la inmersión, la actuación como la transformación son herederos de las posibilidades de placer estético que nos anticipaba la literatura impresa aunque con su especial aporte: la convergencia de la composición literaria, dramática e informática.

\section{6.- ACCIÓN Y NARRACIÓN EN LOS VIDEOJUEGOS EN RED}

La popularización de los juegos multijugadores (multiplayers) ha modificado en gran medida la percepción habitual del videojuego como una actividad solitaria o casi solitaria en la que el jugador se enfrenta a un desafío contra la máquina. En las modalidades multiusuario el sistema informático (computadora o consola + programa de juego) adquiere una naturaleza conceptualmente asimilable a la de un terreno o tablero de juego o a un escenario teatral en el cual dos o más participantes (individualmente o en equipo) realizan sincrónica o asincrónicamente una actividad lúdica, siguiendo reglas y objetivos más o menos cerrados preestablecidos por el programador del juego, cuyo desarrollo definitivo, como en todo juego, dependerá, en última instancia, de las decisiones y acciones de cada uno de los jugadores.

Los juegos en red de simulación relacional generan multitudinarias "comunidades virtuales” (o digitales o simbólicas” que reúnen a cientos de miles de miembros (actores/jugadores) que, con las acciones de los personajes "virtuales" (avatares) que encarnan en el juego, dan lugar a múltiples narraciones de las cuales son protagonistas y coautores, de un modo similar al modo de algunas experiencias de teatro participativo y otras formas escénicas de vanguardia, en la cual el público con sus acciones incide en el desarrollo de la obra. Es el caso del muy publicitado Second Life, y de tantos otros juegos como Ultima Online, Everquest o Colony City.

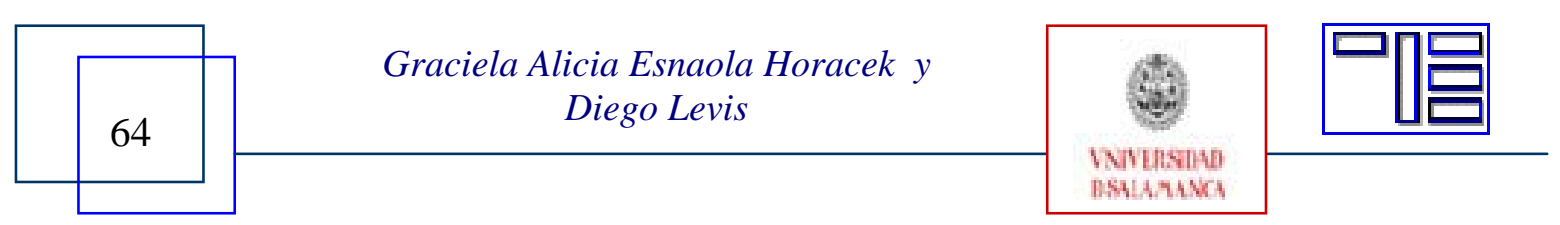


Los miembros de estas comunidades lúdicas pueden jugar de forma individual o colaborativa, formando alianzas temporales o permanentes con otros jugadores. Los juegos en red de este tipo tienen su origen en los juegos de rol (MUD). Los primeros MUD para Arpanet (la red telemática sobre la que se gestó la actual Internet) datan de finales de la década de 1970 y estaban basados en el uso de texto escrito.

La comunidad de jugadores creada alrededor de los juegos de simulación en red opera sobre un espacio simbólico colectivo generando sentimientos de pertenencia en muchos de sus miembros. La "existencia” en el espacio simbólico ("mundo virtual” o "ciberespacio" en la terminología utilizada habitualmente en la literatura sobre el tema) parece, en cierto modo, superponerse a la existencia real de cada uno de los participantes en el juego. En algunos casos, los juegos incorporan traductores automáticos lo que permite que personas con lenguas maternas diferentes y situadas a muchos kilómetros de distancia puedan intercambiar mensajes escritos con relativa fluidez, incrementando las posibilidades relacionales del juego al derribar (al menos parcialmente) las barreras culturales que imponen los idiomas.

Desdeñando el carácter lúdico-creativo que tiene la experiencia de crear y desarrollar personajes (a los que podemos denominar "neoliterarios”) a partir de la imaginación de cada jugador dentro de los mundos de ficción que ofrecen estos juegos de simulación, los editores de estos juegos suelen promocionar sus plataformas como una alternativa válida para vivir una nueva vida "virtual”. La presentación de Ultima on line en el sitio web del juego es un buen ejemplo.

"Un mundo virtual es un lugar en donde usted co-habita con cientos de miles de otras personas simultáneamente. (...) El hecho que usted exista con otra gente real de todo el mundo añade un nivel de inmersión que debe ser experimentada para ser creída (...) Las posibilidades para su nueva vida virtual son realmente infinitas" $"$.

Second Life (Segunda vida) también es muy explícito en su objetivo de crear un mundo y una vida "virtuales" paralelos a la vida en nuestro entorno físico cotidiano. Se trata de una visión limitada y pobre del verdadero potencial y significación sociocultural y educativa de los juegos en red en general y de los juegos de simulación relacional en particular. Los participantes en juegos en red establecen entre sí diversas formas de comunicación. En este proceso resulta primordial el personaje que asume/crea cada uno de los jugadores y las iniciativas que toma durante el juego que determinarán en gran medida el desarrollo de la acción, es decir de la construcción narrativa. Los caracteres de los personajes "protagonizados" en el juego condicionan la forma en la que cada jugador se relaciona con los otros miembros de la comunidad lúdica en la que participa, en particu-

${ }^{1}$ Sitio web de Ultima online: "A Virtual World is a place you co-inhabit with hundreds of thousands of other people simultaneously (...) The fact that you exist with other real people from around the globe adds a level of immersion that must be experienced to be believed". "The possibilities for your new virtual life are truly endless" http://www.ultimaonline.com

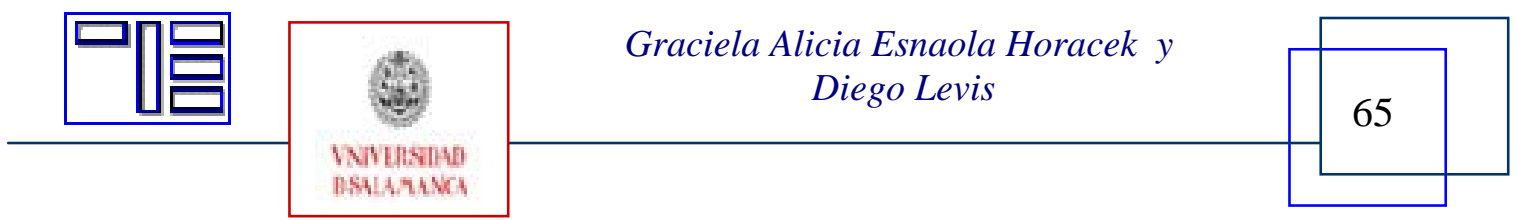


lar en los simuladores relacionales. Personajes moldeados con ingredientes de la personalidad de cada uno de los participantes, de sus deseos y sus pulsiones mezclados con las posibilidades que ofrecen cada uno de los programas utilizados.

El personaje ficcional creado y controlado por cada jugador se incorpora, modificándolo, al escenario narrativo prediseñado por los creadores del juego. Así, sin proponérselo, los jugadores de este tipo de juegos en red participan en la creación de una nueva narración colectiva, hipermedial y neoteatral, en la que se difuminan las fronteras entre autor, actor y espectador. Obras efímeras e improvisadas, sin pretensiones estéticas pero llenas de significación para sus ejecutantes, más interesados en la interacción que en la narración.

\section{6.- BIBLIOGRAFÍA}

BOURDIEU, P. (1990) “Sociología y Cultura”. Editorial Grijalbo, México.

BREMOND, C. (1970) “El mensaje narrativo”. En: La semiología. Bs. As., Tiempo Contemporáneo.

CAILLOIS, R. (1967) Les jeux et les hommes. Le masque et le vertige. Cher: Gallimard

CAMPBELL, J. (1972) The hero with a thousand faces. Mythos Books Princeton University

(1991) The power of myth. Anchor. Ressue Edition

DÉBORD, G. (1995) “La sociedad del espectáculo”. Editorial La Marca. Bs.As.

DE CERTEAU, M. (1996), La invención de lo cotidiano. I. Artes de hacer, México, Universidad Iberoamericana.

ECO, U. (1987) Lector in fabula. La cooperación interpretativa en el texto narrativo. Barcelona: Editorial Lumen.

ESNAOLA HORACEK, G. (2001) El discurso narrativo del relato electrónico. El caso de los videojuegos UNIVERSIDAD DE VALENCIA Fac de Filosofía y CC de la Educación.

ESNAOLA HORACEK, G. (2003) Aprendiendo a leer el mundo del siglo XXI a través de los videojuegos. Propuesta vertebradora desde formación ética y ciudadana para escuelas medias. artículo publicado en el Campus Universitario de Cartuja, Universidad de Granada Seminario Virtual UNESCO

ESNAOLA HORACEK, G. (2004) La construcción de la identidad social y las nuevas tecnologías: un estudio sobre el aprendizaje y los videojuegos en la institución educativa. Tesis de Doctorado publicada por la Universidad de Valencia (Servei de publicaciones)

ESNAOLA HORACEK, G. y SAN MARTÍN ALONSO, A (2005) La identidad en la sociedad de la información: reconstruyendo la fragmentación. UNPA, Universidad de Valencia, en Tecnologias da educação: tecendo relações entre ima-

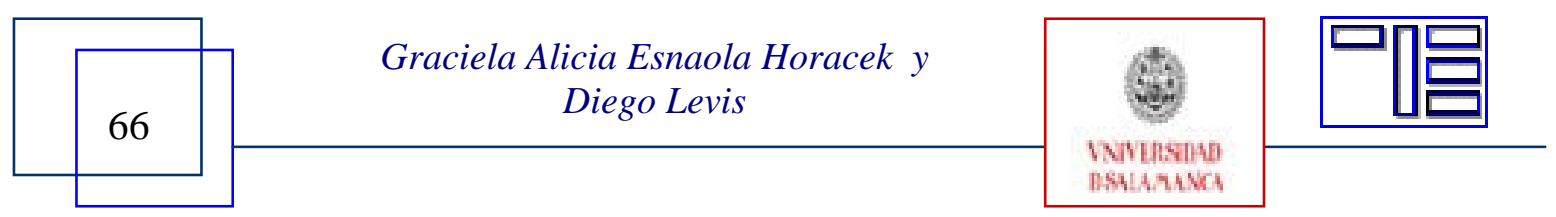


ginário corporeidade e emoções Tecendo relações com as tecnologias da educação Dras Esperon Porto y Vaz Peres (comp). Brasil Edit JM

ESNAOLA HORACEK, G. (2006) Claves culturales en la construcción del conocimiento: ¿qué enseñan los videojuegos? Alfagrama Buenos Aires, 2006/09

FERRÉS, J. “Educar en una cultura del espectáculo”. Paidós. 2000

FRASCA, G.(1997) El videojuego como medio para una ficción interactiva: notas para una poética del joystick. Montevideo: Universidad Catolica del Uruguay, 1997. http://www.orbicom.uqam.ca/english/abstracts/corpus/frasca.html

(1999) Ludology meets narratology Similitude and differences between (video)games and narrative. Paarnasso. Helsinky GEERTZ, C.(2000): La interpretación de las culturas. Gedisa, Barcelona

GROS SALVAT, B.(2000) El ordenador invisible: hacia la apropiación del ordenador en la enseñanza Barcelona : EDIUOC.

(2000) “La dimensión socioeducativa de los videojuegos”. Edutec: Revista electrónica de tecnología educativa, $\mathrm{N}^{\circ} .12$

LANDOW. G (1993) Hypertext. The convergence of Contemporary Critical Theory and Technology. London: The John Hopkins University Press, 1993.

LEVIS, D. (1997): Los videojuegos un fenómeno de masas. Paidós, Barcelona 1997 (1999) La pantalla ubicua, Buenos Aires, CICCUS,

(2005) "Videojuegos y alfabetización digital “ Publicado en “Aula de innovación Educativa”, Barcelona: edit.Grao, n 147, Diciembre de 2005.

(2007) Tecnologías Informáticas en la Educación. A principios del siglo XXI. CABELLO, R. y LEVIS, D (editores)

MASTERMAN, L. (1994) La enseñanza de los medios de comunicación Ediciones de la Torre. Madrid.

MORENO. JULIO (2002) Ser humano. La inconsistencia, los vínculos, la crianza. Zorzal.2002

MORIN, E (2000) La mente bien ordenada (Repensar la forma; reformar el pensamiento), col. en Los tres mundos, Barcelona, Seix-Barral

MURRAY, J. H. (1997). Hamlet on the Holodeck - The Future of Narrative in Cyberspace. Cambridge. The MIT Press., 1997o\#3, Helsinki, 1999.

PÉREZ TORNERO, J. M. (comp.) (2000) “Comunicación y educación en la sociedad de la información”. Nuevos lenguajes y conciencia crítica. Paidós. Barcelona y Buenos Aires

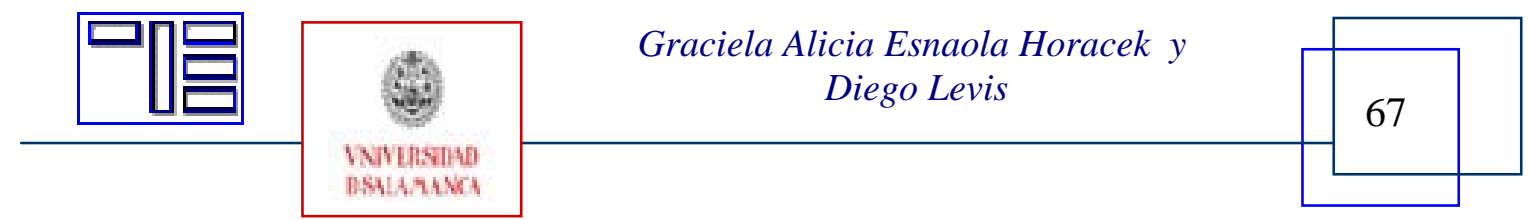


PIAGET, J. (1991) La formación del símbolo en el niño. Imitación, juego y sueño. Imagen y representación. Buenos Aires: Fondo de Cultura Económica.

(1991) La formación del símbolo en el niño. Imitación, juego y sueño. Imagen y representación. Buenos Aires: Fondo de Cultura Económica, 1991

PORUSH, D. and TODD HIVNOR. Génération De Récit Et Théorie Du Jeu : Viktor Shklovsky Et Gameworld. Littérature et informatique : La littérature générée par ordinateur, ?. http://www.refer.org/textinte/littinfo/2_porush.htm

PRINCE, G. (1987). Dictionary of Narratology. Lincoln: University of Nebraska Press.

PROVENZO, E. (1991) Video Kids : Making Sense of Nintendo Harvard University Press

REGUILLO CRUZ, R. (2000) Emergencia de culturas juveniles. Norma, Bogotá

SAN MARTIN ALONSO, A. (2006) Dilema de la relación de las TIC con la escuela. Cuadernos de pedagogía, $\mathrm{N}^{\circ} 363$

QUIROZ, M.T. y TEALDO, A. R. (1996) “Videojuegos o los compañeros virtuales”. Universidad de Lima.

The 1995 GROLIER Multimedia Encyclopedia. Danbury: Grolier Electronic Publishing, Inc., 1995.

TURKLE SHERRY (2005) The second self: computer and human spirits. Cambridge. Mit Press

(1997) Life on the sreen: Identity in the age of Internet.New York Touch Stone

VIDART, D. (1995). El juego y la condición humana. Notas para una antropología de la libertad en la necesidad. Montevideo: Ediciones de la Banda Oriental.

Para citar la presente editorial puede utilizar la siguiente referencia:

ESNAOLA HORACEK, Graciela A. y LEVIS, D. (2008). La narrativa en los videojuegos: Un espacio cultural de aprendizaje socioemocional. En SÁNCHEZ i PERIS, Francesc J. (Coord.) Videojuegos: una herramienta educativa del "homo digitalis" [monográfico en línea]. Revista Electrónica Teoría de la Educación: Educación y Cultura en la Sociedad de la Información. Vol. 9, n 3. Universidad de Salamanca [Fecha de consulta: $\mathrm{dd} / \mathrm{mm} / \mathrm{aaaa}]$.

http://www.usal.es/ teoriaeducacion/rev_numero_09_03/n9_03_esnaola_levis.pdf ISSN: 1138-9737
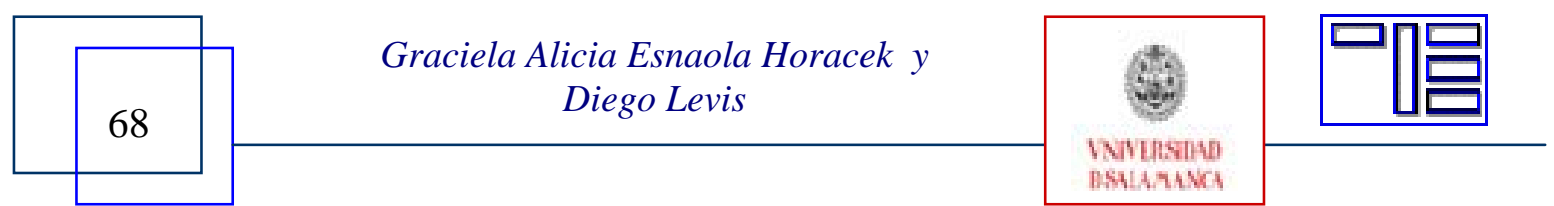